\title{
Design of A Low Profile Array Transducer in d15 Mode for High Angled Shear Wave Generation
}

\author{
Zhen Qiu, Richard O’Leary, Anthony Gachagan \\ Centre for Ultrasonic Engineering, \\ University of Strathclyde, \\ Glasgow, UK \\ zhen.qiu@strath.ac.uk
}

\begin{abstract}
Shear wave inspection is generated from mode conversion of longitudinal waves, using a selected, angled wedge positioned between the transducer and the test specimen. However, in certain scenarios where access is restricted the combination of the transducer and the wedge can be too cumbersome for in situ deployment. In this work, a low profile, linear ultrasound array transducer is proposed to generate shear waves via direct coupling to the component surface precluding the requirement for a wedge. The array transducer was designed using finite element modelling, and a prototyped array was manufactured with 32 elements and operating frequency at $\sim 2 \mathrm{MHz}$. Preliminary imaging results has shown the shear wave beam generated can be operated at high angles up to $80^{\circ}$.
\end{abstract}

Keywords-shear wave; direct coupling; linear array; low profile; high angle; NDT/E;

\section{INTRODUCTION}

Since Sproule developed the first shear wave angle probes in 1947, shear wave inspection has been developed as a common technique within NDE for many types of discontinuities across many industrial sectors [1]. Shear wave operation has shorter wavelength compared to longitudinal waves and thus leads to improved minimum flaw size resolution. Conventionally, shear wave generation is achieved by mode conversion and Snell's Law using an angled wedge positioned between the transducer and the specimen [2]. The selection of wedges is specified by the material of test specimen and desired beam angle, and thus multiple wedges would be required if inspections needs to carried out from different angles. Moreover, the combination of the array and the wedge can be too cumbersome to deploy in certain scenarios where access is restricted, limited height for example; the examination volume might also be limited due to the access offset caused by the size of the wedge. Although a high angle beam can be generated using phased array probes, the standard design principle, element pitch less then half wavelength based on longitudinal wavelength, typically leads to the generation of side lobes or grating lobes for shear wave when the angle is higher than $45^{\circ}$, obscuring the return echoes resulting in difficulties analyzing the resultant ultrasonic signals [3].

Most research on shear wave inspection has been focused on guided wave in plate-like structures. In addition to the

\author{
Jeff Dobson ${ }^{1,2}$ \\ ${ }^{1}$ Centre for Ultrasonic Engineering, \\ University of Strathclyde, \\ Glasgow, UK \\ ${ }^{2}$ PZFlex, Thornton Tomasetti, \\ Glasgow, UK
}

electro-magnetic acoustic transducer (EMAT) [4] and flexible PVDF transducer [5] to generate shear waves, piezoelectric transducers in different modes of oscillation have gained increasing interests because of the development of ferroelastric domain engineering. Thickness-shear type piezoelectric ceramic associated with the $d_{15}$ coupling coefficient [6][7] has its poling direction, referred to as the $3^{\text {rd }}$ axis, tangential to the electrical excitation direction, $1^{\text {st }}$ axis. Applying a voltage to electrodes placed across the $1^{\text {st }}$ axis will induce a pure torsional displacement around $2^{\text {nd }}$ axis. This displacement transmits a shear wave into the test component through a viscous couplant. The piezo deformations induced by $d_{15}$ and $d_{33}$, the common thickness-extensional type, are presented in Fig. 1. A new face-shear $\left(d_{36}\right)$ mode was reported in PMN-PT piezocrystals [8] which is superior to $d_{15}$ mode has been applied to NDE field as well [9], however its applications is limited due to its less stable domains and high cost. This face-shear mode has also recently been realized in both soft and hard PZT ceramics [10] and multiple modes of guided waves were generated and detected. However, these materials are not yet commercially available.
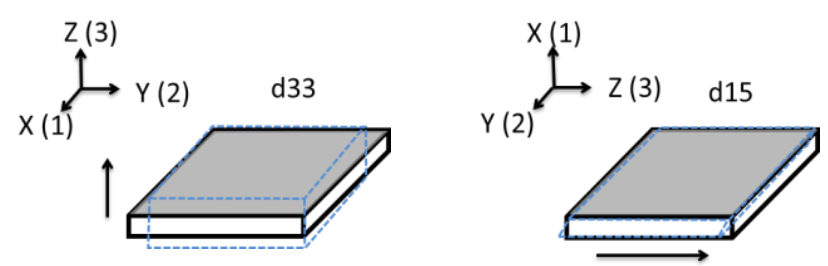

Fig. 1Piezo deformation induced by d 33 and d 15 coefficients. Arrow indicates the poling direction

In this work, the authors developed a linear shear array transducer using commercially available $d_{15}$ piezoelectric ceramic material. The array transducer aims to generate high angled shear wave via direct coupling to the component surface, with low profile in both height and size and no requirement for an additional wedge. As a preliminary feasibility study, only bulk shear wave generation in thick test specimens has been considered at this stage.

\section{MATERIAL AND METHOD'S}

Soft piezoceramic PZ27 $d_{15}$ plates (Meggitt-Ferroperm, Denmark) were available for application in the proposed 
array. With a geometry of $14 \times 7 \times 1 \mathrm{~mm}$, the plates were poled along the $7 \mathrm{~mm}$ side direction and electrodes were placed on $14 \times 7 \mathrm{~mm}$ faces. The problem space of developing the shear array transducer was explored using finite element modelling package, PZFlex (Thornton Tomasetti, Cupertino, CA). The operational frequency was chosen to be $2 \mathrm{MHz}$ to allow further comparison with an existing commercial standard linear array. Single array element was assessed firstly with free boundary condition and then damped with backing material and coupled to a steel block. Maximizing shear energy generation, minimizing inter-element crosstalk, and minimizing side / grating lobes during steering were considered as design criteria of the shear array.

Array performance was assessed by considering the FE derived shear energy distribution field using the square root of the second invariant of deviatoric stress tensor. This allowed the beam profiles, array's focusing and steering abilities to be evaluated. To assess defect interaction, a cluster of voids with diameter of 1 wavelength of shear wave were created in the model to present porous defects in welds. A transmit-receive circuit switch was built into the model to simulate the related electronics for imaging operation. The FE model was used to create the full-matrix capture (FMC) dataset of the array in order to generate total focusing method (TFM) images [11]. This allowed for facile comparison of the proposed array structure with a conventional thickness mode array of comparable frequency of operation. An initial prototype shear wave array device has also been fabricated and preliminary experimental results previewed.

\section{RESULTS}

\section{A. Array element geometry}

Admittance / Impedance spectra of array elements with varying geometries were plotted to identify the desired resonance characteristics. With element length to be fixed at $14 \mathrm{~mm}$ as per material provided, element thickness and the width along poling direction were varied and investigated. Fig. 2 (a) presents the admittance spectra of un-clamped elements as an example, with a thickness of $0.45 \mathrm{~mm}$, a length of $14 \mathrm{~mm}$, and width varied from $0.4 \mathrm{~mm}$ to $2 \mathrm{~mm}$ with 0.1 $\mathrm{mm}$ step, which equals a width to thickness ratio (W/Th) from 0.9 to 4.5 . Each peak in the admittance spectrum represents a resonance. As can be seen from the figure, a larger W/Th correlates with a stronger and more distinct shear resonance. However, large W/Th ratio is not practical for array element design due to the conventional $\lambda / 2$ array design principle. Since the desired frequency was selected to be $2 \mathrm{MHz}$, the final selection of element geometry was made between $14 \mathrm{x}$ $0.7 \times 0.45 \mathrm{~mm}$ and $14 \times 0.6 \times 0.5 \mathrm{~mm}$. By comparing their shear energy distribution in steel block at $5 \mathrm{~mm}$ depth, it was found that the former produced 1.4 times more shear energy than the latter. The array element was therefore determined to be $14 \times 0.7 \times 0.45 \mathrm{~mm}$ for higher shear energy generation. Its impedance spectrum is plotted in Fig. 2 (b) with two resonances near $2 \mathrm{MHz}$ and $3 \mathrm{MHz}$, respectively. To further identify the shear vibration at resonances, the mode shapes at both resonances were plotted in the form of displacement along poling direction, as shown in Fig. 3.
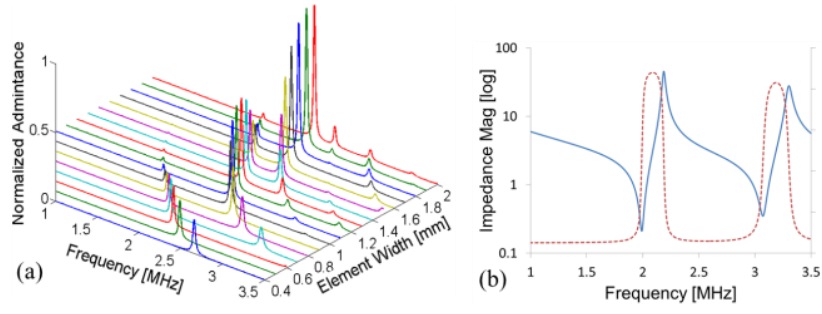

Fig. 2 (a) Admittance spectra of element of $0.45 \mathrm{~mm}$ thick, $14 \mathrm{~mm}$ in length, and with varying widths (b) impedance spectrum of element: $0.7 \mathrm{~mm}$ in width, with two resonances

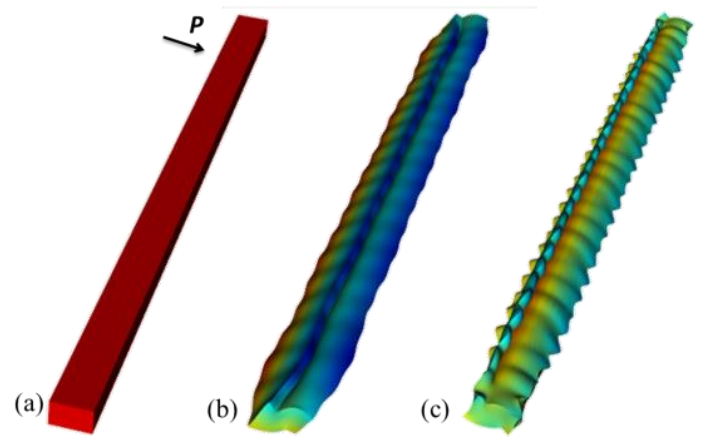

Fig. 3 Displacement of array element in poling direction $P$ with free boundary condition in FEM (a) array element geometry (b) displacement plotted on mode shape at $1^{\text {st }}$ resonance at $2 \mathrm{MHz}$ (c) $2^{\text {nd }}$ resonance at 3.1 $\mathrm{MHz}$.

Although the shear mode at $2 \mathrm{MHz}$ resonance coupled with other vibration modes as presented, this is expected and hard to avoid as the selected geometry doesn't have the ideal W/Th ratio defined by IEEE standard, which should be at least 10:1 for uniform thickness-shear resonance [12]. The energy distribution field generated by this single element operating into a steel load is plotted in Fig. 4 and shows that not only shear wave was generated and propagated within the steel specimen, longitudinal waves are generated as well, with lower amplitude, $-16.5 \mathrm{~dB}$ when compared to the shear wave peak amplitude.

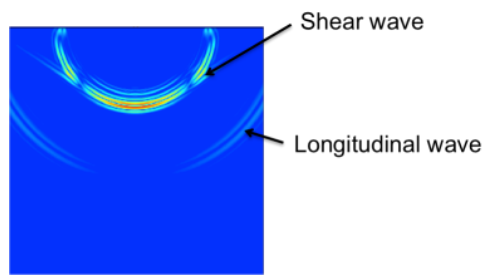

Fig. 4 Energy distribution field presents both primary shear wave and secondary longitudinal wave were generated by the array element.

\section{B. Array transducer assessment}

With the element geometry determined, the separation space between elements was investigated. A trade-off decision has to be made between minimizing inter-element cross talk and minimizing the side lobes/grating lobes. To this end, an array model was constructed in PZFlex with five array elements placed in a linear configuration and backed with $10 \%$ v/v tungsten loaded hard-set epoxy (CY1301/HY1300). One outside element was excited and the displacement across 
the model surface was recorded along the poling direction of the piezoelectric material. Table 1 lists the displacement in $\mathrm{dB}$ recorded at the surface of the most adjacent element to the excited element, with $0.1,0.2$, and $0.3 \mathrm{~mm}$ element spacing, respectively. Although $0.3 \mathrm{~mm}$ separation appears to have resulted in minimum cross talk effect, it resulted in a corresponding increase in amplitude of side lobes in the energy field. The intermediate $0.2 \mathrm{~mm}$ kerf was therefore selected for the proposed array configuration. The final design of the array parameters are listed in Table 2.

TABLE I. SURFACE DISPLACEMENT (dB) AT NEAREST ADJACENT ELEMENT WITH VARYING ELEMENT SPACING

\begin{tabular}{|c|r|r|r|}
\hline & \multicolumn{3}{|c|}{ Element Spacing (mm) } \\
\hline \multirow{2}{*}{$\begin{array}{c}\text { Displacement (dB) in poling } \\
\text { direction }\end{array}$} & $\mathbf{0 . 1}$ & $\mathbf{0 . 2}$ & $\mathbf{0 . 3}$ \\
\cline { 2 - 4 } & -10.1 & -11.0 & -12.5 \\
\hline
\end{tabular}

In order to investigate the imaging performance of the shear array, a 32-element array model was built in PZFlex using the characteristics listed in Table II. Time delays were applied to individual elements for beam focusing and steering. Fig. 5 presents the shear energy distribution map representing the beam profile of the array when the beam is focused and steered at different angles in the range 45-80 degrees. No grating lobes were observed during steering and the intensity of side lobes were all below $-24 \mathrm{~dB}$. When the steering angle was as high as 80 degrees, strong response was observed, -9 $\mathrm{dB}$ of peak magnitude, which is attributed to a surface wave propagating along the specimen - this effectively limits the predicted steering capability of the current array configuration to 80 degrees.

\section{TABLE II. LISTS OF ARRAY CHARACTERISTICS}

\begin{tabular}{|c|c|}
\hline Frequency & $2 \mathrm{MHz}$ \\
\hline No of elements & 32 \\
\hline Primanry asix pitch & $0.9 \mathrm{~mm}$ \\
\hline Inter-element spacing & $0.2 \mathrm{~mm}$ \\
\hline Width of element & $14 \mathrm{~mm}$ \\
\hline Total active length & $28.6 \mathrm{~mm}$ \\
\hline Backing & $10 \% \mathrm{v} / \mathrm{v}$ Tungsten loaded hard set epoxy \\
\hline
\end{tabular}

To assess the array's imaging ability within the FEM package, FMC and TFM were applied to look at defects in a steel loaded coupled to the array. Although the dynamic focusing imaging method would be more appropriate for this shear array application, it represents poor computational efficiency to generate an image with adequate resolution.

The defective area in the steel load comprised three side drilled holes $30 \mathrm{~mm}$ from the array front face and directly beneath array central axis. This is to represent a simple porous defect. Each void had diameter of 1 shear wave wavelength, with a 1.5 shear wave wavelength separation distance. For comparison purpose, a similar model was built with a longitudinal array, with array characteristics changed accordingly to keep operational frequency and active array aperture equivalent. Fig. 6 illustrates a schematic of the 32-element model with void defects. The TFM imaging results from shear array and longitudinal array are also presented. The shear array demonstrates higher resolution imaging potential with the porous defects accurately localized and a sizing error of between $5 \%-15 \%$ of the defects' actual size.
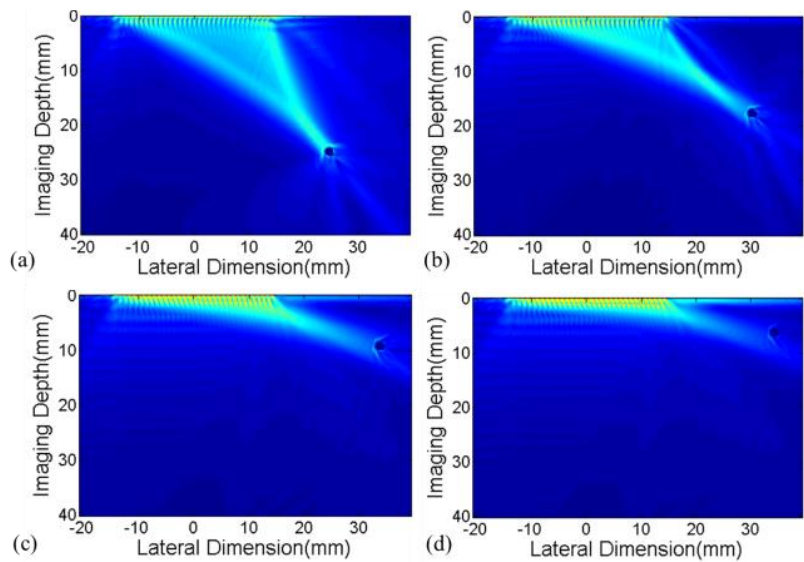

Fig. 5 FE derived shear energy distribution map when beam steered at (a) 45 degree; (b) 60 degree; (c) 75 degree and (d) 80 degree with defect located at $35 \mathrm{~mm}$ away from the centre of the array

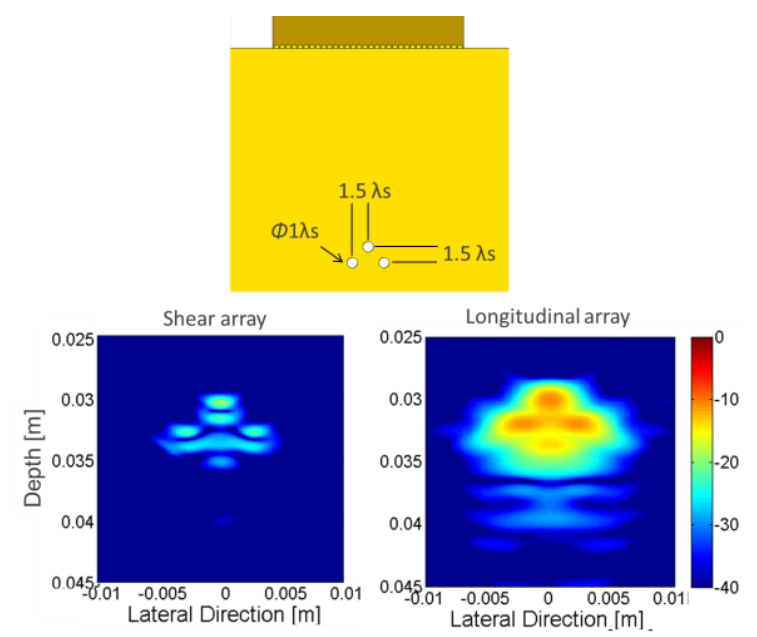

Fig. 6 TFM images of same sized defects obtained by shear array and longitudinal array.

\section{Array fabrication}

A prototype shear array was manufactured. Due to the size of the available material plates, five plates were required to be grouped together to build one 32-element array. Each of the five plates were lapped individually and diced to specific widths in order to assemble the proposed array configuration. The plates were aligned and secured on dicing tape and a specific designed flexible PCB was glued to the edge of the plates along the array's primary axis, using cyano-acrylate adhesive and silver loaded epoxy (G3349, Agar Scientific, UK). After the silver loaded epoxy was cured, the assembly was diced through the PZT, the loaded epoxy and section of the flexible PCB to mechanically and electrically isolate each element. A similar fabrication process was reported in [13].

The assembly was then transferred into aluminum housing and $10 \% \mathrm{v} / \mathrm{v}$ tungsten loaded epoxy mixture was carefully 
filled from the rear opening of the case to act as both backing material and kerf filler. Fig. 7 presents the photographs of the finished device, with its front face shown in the inset prior to the common ground electrode being applied. The overall height of the transducer was $15 \mathrm{~mm}$. Because of the effect caused by backing layer and the lapping processes, the resonance frequency has shifted slightly towards to the lower end: the resulting frequency of array elements is $1.78 \pm 0.08$ $\mathrm{MHz}$ at electrical resonance and $2.28 \pm 0.04 \mathrm{MHz}$ at its mechanical resonance.

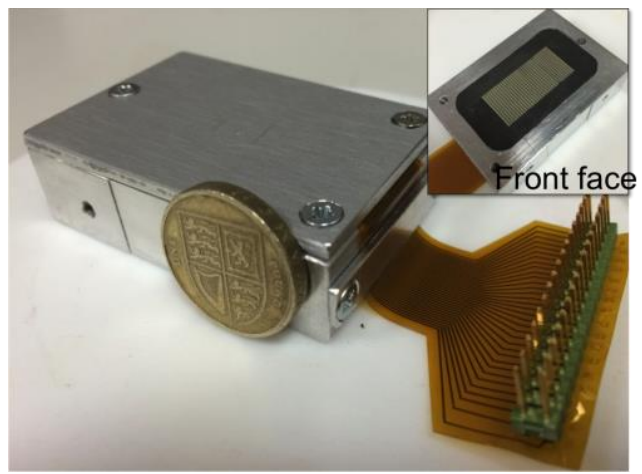

Fig. 7 Picture of the array prototype

\section{Array experimental results}

The manufactured shear array was tested using a $50 \mathrm{~mm}$ thick calibration-test block with a curved edge as shown in Fig. 8 (a). Transducer coupling to the steel surface was achieved using a commercial normal incidence shear gel (Magnaflux, Glenview, USA). The array was driven by Dynaray array controller (Zetec Inc.USA), with $40 \mathrm{~V}$ pulse and $35 \mathrm{~dB}$ gain in reception. The ultrasound beam was focused at $50 \mathrm{~mm}$ distance and the curved edge of the testing block was used as the imaging target. The beam was then steered between angles -10 to 80 degree with a step of 1 degree and Fig.8 (b) illustrates the resultant sector B-scan. The curved edge is generally visible in the image within the overall steering range. A strong near field reverberation beneath the center of the array is evident, particularly in the region with low steering angle, i.e. -10 to 10 degree range. Further investigation on the cause of this will be carried out in the near future.
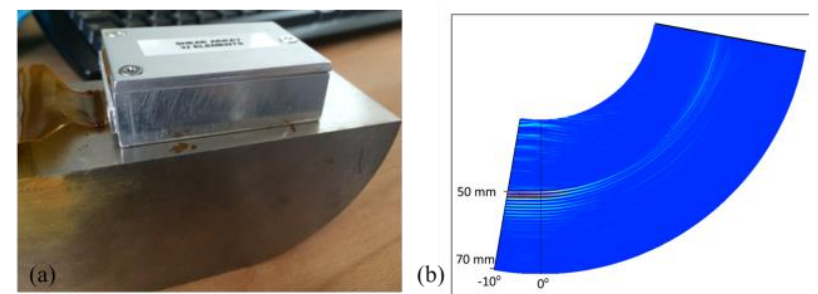

Fig. 8 (a) Experimental setup of the array with a calibration-test steel block (b) image of curved edge of the testing block

\section{CONCLUTIION}

In this work, thickness-shear $\left(d_{15}\right)$ mode of piezoelectric ceramic material was used to build a linear array transducer, which makes use of the shear motion of its elements to generate a shear wave directly coupled into a test specimen.
The fact that no requirement of additional wedge allows its usage in the scenarios where inspection access is limited. The $2 \mathrm{MHz}, 32$-element array was designed using finite element modelling, with low inter-element crosstalk $(<-10 \mathrm{~dB})$ and side/grating lobes $(<-24 \mathrm{~dB})$. The array's steering and imaging ability were assessed with modelling as well, and the preliminary experimental imaging result indicating that the generated shear wave beam can be swept at high angles up to $80^{\circ}$ using the designed configuration.

\section{ACKNOWLEDGMENTS}

This work has been carried out under the auspices of the UK Research Centre for NDE (RCNDE) (EPSRC Grant EP/L022125/1).

\section{REFERENCES}

[1] C. J. Hellier, Handbook of Nondestructive Evaluation, vol. 69. The McGraw_Hill Companies, Inc, 2012.

[2] J. L. Rose, Ultrasonic Waves in Solid Media. Cambridge University Press, 2004.

[3] B. W. Drinkwater and P. D. Wilcox, NDT E Int., vol. 39, no. 7, pp. 525-541, Oct. 2006.

[4] R. Ribichini, F. Cegla, P. B. Nagy, and P. Cawley, IEEE Trans. Ultrason. Ferroelectr. Freq. Control, vol. 58, no. 12, pp. 2571-2581, 2011.

[5] T. R. Hay and J. L. Rose, Sensors Actuators A Phys., vol. 100, no. 1, pp. 18-23, Aug. 2002.

[6] A. Kamal and V. Giurgiutiu, Smart Mater. Struct., vol. 23, no. 8, p. 085019, Aug. 2014.

[7] G. Boivin, M. Viens, and P. Belanger, AIP Conf. Proc. 1706, vol. 030019, p. 030019, 2016.

[8] P. Han, W. Yan, J. Tian, X. Huang, and H. Pan, Appl. Phys. Lett., vol. 86 , no. 5, pp. 1-3, 2005.

[9] W. Zhou, H. Li, and F.-G. Yuan, Ultrasonics, vol. 57, pp. 135-143, 2015.

[10] H. Miao, X. Chen, H. Cai, and F. Li, J. Appl. Phys., vol. 118, no. 21, p. 214102, 2015.

[11] C. Holmes, B. W. Drinkwater, and P. D. Wilcox, NDT E Int., vol. 38, no. 8, pp. 701-711, Dec. 2005.

[12] "An American National Standard IEEE Standard on Piezoelectricity," ANSI/IEEE STd 176-1987, 1988.

[13] P. Glynne-Jones, C. E. M. Demore, C Ye, Y Qiu, S. Cochran, and M. Hill, IEEE Trans. Ultrason. Ferroelectr. Freq. Control, vol. 59, no. 6, pp. 1258-1266, Jun. 2012. 